Jour. of Phys. A, 31, N1, (1998),L39-L44.

\title{
FORMULA FOR THE RADIUS OF THE SUPPORT OF THE POTENTIAL IN TERMS OF SCATTERING DATA
}

\author{
A. G. RAMm \\ Department of Mathematics, Kansas State University, \\ Manhattan, KS 66506-2602, USA \\ J. H. Arredondo And B. C. Izquierdo \\ Department of Mathematics, University Autonoma Metropolitana, \\ Av.Michoacan y Purisima, Iztapalapa, Apartado Post. 55-532, C.P.09340 \\ Mexico City D.F., Mexico
}

\begin{abstract}
Let $q(r), r=|x|, x \in \mathbb{R}^{3}$, be a real-valued square-integrable compactly supported function, and $[0, a]$ be the smallest interval containing the support of $q(r)$. Let $A\left(\alpha^{\prime}, \alpha\right)=A\left(\alpha^{\prime} \cdot \alpha\right)$ be the corresponding scattering amplitude at a fixed positive energy, $k^{2}=1$. Let $\delta_{\ell}$ be the phase shifts at $k=1$. It is proved that $\lim _{\ell \rightarrow \infty}\left(\frac{2 \ell+1}{e}\left|\delta_{\ell}\right|^{\frac{1}{2 \ell}}\right)=a$, provided that $q(r)$ does not change sign in some, arbitrary small, neighborhood of $a$.
\end{abstract}

1. Introduction. The aim of this paper is to give a partial justification of the modified conjecture due to A.G. Ramm [R1, p.356, formula (7)].

Let us make the following assumption.

Assumption (A):

the potential $q(r), r=|x|$, is spherically symmetric, real-valued, $\int_{0}^{a}|q|^{2} d r<\infty$, and $q(r)=0$ for $r>a$, but $q(r) \neq 0$ on $(a-\varepsilon, a)$ for all sufficiently small $\varepsilon>0$.

The number $a>0$ we call the radius of compactness of the potential, or simply the radius of the potential. Let $A\left(\alpha^{\prime}, \alpha\right)$ denote the scattering amplitude corresponding to the potential $q$ at a fixed energy $k^{2}>0$. Without loss of generality let us take $k=1$ in what follows. By $\alpha^{\prime}, \alpha \in S^{2}$ the unit vectors in the direction of the scattered, respectively, incident wave, are meant, $S^{2}$ is the unit sphere in $\mathbb{R}^{3}$. Let us write, as in $[R 1]$,

$$
A\left(\alpha^{\prime}, \alpha\right)=\sum_{\ell=0}^{\infty} A_{\ell}(\alpha) Y_{\ell}\left(\alpha^{\prime}\right)
$$

where $Y_{\ell}=Y_{\ell m},-\ell \leq m \leq \ell$, are the usual orthonormal in $L^{2}\left(S^{2}\right)$ spherical harmonics (defined by formula (10) in [R1, p.21]) and $A_{\ell}:=A_{\ell m}$ are the Fourier coefficients of $A$ :

$$
A_{\ell}(\alpha)=\left(A\left(\alpha^{\prime}, \alpha\right), Y_{\ell m}\left(\alpha^{\prime}\right)\right)_{L^{2}\left(S^{2}\right)}
$$

1994 PACS 03.65.NK, 03.80.+r.

1991 Mathematics Subject Classification. 35R30, 81F05, 86A15.

Key words and phrases. Scattering, radius of the support of the potential.

Typeset by $\mathcal{A} \mathcal{M} \mathcal{S}-\mathrm{TEX}_{\mathrm{E}}$ 
It is of interest to obtain any information about $q$ from the (fixed-energy) scattering data, that is, from the scattering amplitude $A\left(\alpha^{\prime}, \alpha\right)$, or, equivalently, from the coefficients $A_{\ell}(\alpha)$. Very few results of such type are known, although it was proved by A.G. Ramm that $q(x)$ is uniquely defined by the values $A\left(\alpha^{\prime}, \alpha\right)$ [R1, p.64], [R3], [R4], [R7].

The only other result of such type, known to the authors, is the necessary and sufficient condition for $q(x)=q(|x|)$; it was proved by A.G. Ramm [R1, p.131], that $q(x)=q(|x|)$ if and only if $A\left(\alpha^{\prime}, \alpha\right)=A\left(\alpha^{\prime} \cdot \alpha\right)$. Of course, the necessity of this condition was a common knowledge, but the sufficiency, that is, the implication: $A\left(\alpha^{\prime}, \alpha\right)=A\left(\alpha^{\prime} \cdot \alpha\right) \Rightarrow q(x)=q(|x|)$, is a recent result [R2].

A modified conjecture from [R1, p.356] (the exponent in [R1] was $\frac{1}{\ell}$ while in formula (1.3) below it is $\frac{1}{2 \ell}$ ) says that if the potential $q(x)$ is compactly supported, and $a>0$ is its radius (defined for non-spherically symmetric potentials in the same way as for the spherically symmetric), then

$$
a=\varlimsup_{\ell \rightarrow \infty}\left(\frac{2 \ell+1}{e}\left[\sup _{\substack{\alpha \in S^{2} \\-\ell \leq m \leq \ell}}\left|A_{\ell m}(\alpha)\right|\right]^{\frac{1}{2 \ell}}\right)
$$

In this paper we prove (1.3) for the spherically symmetric potentials $q=q(r)$.

If $q=q(r)$ then

$$
A_{\ell m}(\alpha)=\tilde{a}_{\ell} Y_{\ell m}(\alpha)
$$

where $\tilde{a}_{\ell}$ depends only on $\ell$ and $k$, but not on $\alpha$ or $\alpha^{\prime}$. Since $k=1$ is fixed, $\tilde{a}_{\ell}$ depends only on $\ell$ for $q=q(r)$. To prove (1.4) assuming $q=q(r)$, one takes $A\left(\alpha^{\prime}, \alpha\right)=A\left(\alpha^{\prime} \cdot \alpha\right)$ and calculates

$$
A_{\ell m}(\alpha)=\int_{S^{2}} A\left(\alpha^{\prime} \cdot \alpha\right) \overline{Y_{\ell m}\left(\alpha^{\prime}\right)} d \alpha^{\prime}=\tilde{a}_{\ell} \overline{Y_{\ell m}(\alpha)}
$$

where

$$
\tilde{a}_{\ell}:=\frac{2 \pi}{C_{\ell}^{\left(\frac{1}{2}\right)}(1)} \int_{-1}^{1} A(t) C_{\ell}^{\left(\frac{1}{2}\right)}(t) d t, \quad \ell=0,1,2, \ldots
$$

Here we have used formula (14.4.46) in [RK, p.413], and $C_{\ell}^{(p)}(t)$ are the Gegenbauer polynomials (see [RK, p.408]). Since $C_{\ell}^{\left(\frac{1}{2}\right)}=P_{\ell}(t), P_{\ell}(1)=1$, where $P_{\ell}(t)$ are the Legendre polynomials (see, e.g., [RK, p.409]), formula (1.6) can be written as:

$$
\tilde{a}_{\ell}=2 \pi \int_{-1}^{1} A(t) P_{\ell}(t) d t .
$$

Formula (1.3) for $q=q(r)$ can be written as

$$
a=\varlimsup_{\ell \rightarrow \infty}\left(\frac{2 \ell+1}{e}\left|\tilde{a}_{\ell}\right|^{\frac{1}{2 \ell}}\right) .
$$

Indeed,

$$
\sup _{\substack{\alpha \in S^{2} \\-\ell \leq m \leq \ell}}\left|Y_{\ell m}\right|=O\left(\ell^{\frac{1}{2}}\right)
$$


as is well known (see, e.g., [MP, p.261]). Thus

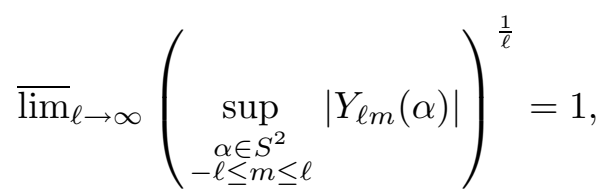

and formula (1.8) follows from (1.3), (1.4) and (1.9). Formula (1.8) can be rewritten in the equivalent form:

$$
a=\frac{2}{e} \varlimsup_{\ell \rightarrow \infty}\left(\ell\left|\tilde{a}_{\ell}\right|^{\frac{1}{2 \ell}}\right)
$$

Note that assumption (A), made in the Introduction, implies the following assumption:

Assumption $\left(\mathrm{A}^{\prime}\right)$ : the potential $q(r)$ does not change sign in some left neighborhood of the point $a$.

This assumption in practice is not restrictive, however, as shown in [R1,p.282], the potentials which oscillate infinitely often in a neighborhood of the right end of their support, may have some new properties which the potentials without this property do not have. For example, it is proved in $[R 1, p .282]$, that such infinitely oscillating potentials may have infinitely many purely imaginary resonances, while the potentials which do not change sign in a neighborhood of the right end of their support cannot have infinitely many purely imaginary resonances. Therefore it is of interest to find out if assumption A' is necessary for the validity of (1.10).

The main result of this paper is:

Theorem 1.1. Let assumption (A) hold. Then formula (1.10) holds with $\overline{\lim }$ replaced by $\lim$.

This result can be stated equivalently in terms of the phase shift $\delta_{\ell}$ defined in formula (3.2) of Section 3. Namely:

$$
\lim _{\ell \rightarrow \infty}\left(\frac{2 \ell+1}{e}\left|\delta_{\ell}\right|^{\frac{1}{2 \ell}}\right)=a
$$

In Section 2 we prove an auxiliary result:

Lemma 1.1. If $q=q(r) \in L^{2}(0, \infty), q(r)$ is real-valued and does not change sign in some interval $\left(a_{1}, a\right]$ where $a_{1}<a$, and $a$ is the radius of $q$, then

$$
a=\lim _{m \rightarrow \infty}\left|\int_{0}^{\infty} q(r) r^{m} d r\right|^{\frac{1}{m}}, m=1,2, \ldots
$$

In Section 3 we prove (1.10') and, therefore, formula (1.3) for spherically symmetric potentials.

2. Proof of Lemma 1.1. First, we obtain a slightly different result than (1.11) as an immediate consequence of the Paley-Wiener theorem. Namely, we prove Lemma 1.1 with a continuous parameter $t$ replacing the integer $m$ and $\overline{\lim }$ replacing $\lim$. This is done for $q(r) \in L^{2}(0, a)$ and without additional assumptions about $q$. However, we are not able to prove Lemma 1.1 assuming only that $q(r) \in L^{2}(0, a)$.

Since $q(r)$ is compactly supported, one can write

$$
I(t):=\int_{0}^{\infty} q(r) r^{t} d r=\int_{0}^{a} q(r) e^{t \ln r} d r=\int_{-\infty}^{\ln a} q\left(e^{u}\right) e^{u} e^{t u} d u .
$$

Let us recall that Paley-Wiener theorem implies the following claim (see $[\mathrm{L}]$ ): 
If $f(z)=\int_{b_{1}}^{b_{2}} g(u) e^{-i u z} d u,\left[b_{1}, b_{2}\right]$ is the smallest interval containing the support of $g(u)$, and $g(u) \in L^{2}\left(b_{1}, b_{2}\right)$, then

$$
b_{2}=\varlimsup_{t \rightarrow+\infty}\left(t^{-1} \ln |f(i t)|\right)=\varlimsup_{t \rightarrow+\infty} \frac{\ln \left|\int_{b_{1}}^{b_{2}} g(u) e^{t u} d u\right|}{t} .
$$

Thus, using (2.1) and (2.2), one gets:

$$
\ln a=\varlimsup_{t \rightarrow+\infty}\left(t^{-1} \ln \left|\int_{-\infty}^{\ln a} q\left(e^{u}\right) e^{u} e^{t u} d u\right|\right)
$$

Formula (2.3) is similar to (1.11) with $m$ replaced by $t$ and lim replaced by $\overline{\lim }$.

Remark 2.1. We have used formula (2.2) with $b_{1}=-\infty$, while in the Paley-Wiener theorem it is assumed that $b_{1}>-\infty$. However, for $b_{1}<b_{2}, g \not \equiv 0$ on $\left[b_{2}-\varepsilon, b_{2}\right]$ for any $\varepsilon>0$, one has:

$$
\int_{-\infty}^{b_{2}} g(u) e^{t u} d u=\int_{-\infty}^{b_{1}} g(u) e^{t u} d u+\int_{b_{1}}^{b_{2}} g(u) e^{t u} d u:=h_{1}(t)+h_{2}(t) .
$$

Thus $\lim _{t \rightarrow \infty} \frac{h_{1}(t)}{h_{2}(t)}=0$, and

$$
\varlimsup_{t \rightarrow \infty} \frac{\ln \left|h_{1}(t)+h_{2}(t)\right|}{t}=\varlimsup_{t \rightarrow \infty} \frac{\ln \left|h_{2}(t)\right|}{t}+\lim _{t \rightarrow \infty} \frac{\ln |1+o(1)|}{t}=\varlimsup_{t \rightarrow \infty} \frac{\ln \left|h_{2}(t)\right|}{t}=\ln a .
$$

Therefore formula (2.3) follows.

To prove (1.11), we use a different approach independent of the Paley-Wiener theorem. We will use (1.11) below, in formula (3.15). In this formula the role of $q(r)$ in (1.11) is played by $r q(r)[1+\epsilon(r, \ell)]$, where $\epsilon=O\left(\frac{1}{\ell}\right)$. Let us prove (1.11).

Assume without loss of generality that $q \geq 0$ near $a$. Let $I:=\int_{0}^{a} q(r) r^{m} d r=\int_{0}^{a_{1}} q(r) r^{m} d r+$ $\int_{a_{1}}^{a} q(r) r^{m} d r:=I_{1}+I_{2}$. We have $\left|I_{1}\right|<c a_{1}^{m}, c_{1}(a-\eta)^{m}<I_{2}<c_{2} a^{m}$, where $\eta$ is an arbitrary small positive number. Thus, $I>0$ for all sufficiently large $m$, and $I^{1 / m}=I_{2}^{1 / m}\left(1+\frac{I_{1}}{I_{2}}\right)^{1 / m}$. One has $a-\eta \leq I_{2}^{1 / m} \leq a$ and $\frac{I_{1}}{I_{2}} \rightarrow 0$ as $m \rightarrow \infty$. Since $\eta$ is arbitrary small, it follows that $\lim _{m \rightarrow \infty} I^{1 / m}=a$. This completes the proof of (1.11). Lemma 1.1 is proved.

3. Proof of formula (1.10). By (1.4) and (1.5), one has

$$
A\left(\alpha^{\prime} \cdot \alpha\right)=\sum_{\ell=0}^{\infty} \tilde{a}_{\ell} \overline{Y_{\ell}(\alpha)} Y_{\ell}\left(\alpha^{\prime}\right):=4 \pi \sum_{\ell=0}^{\infty} a_{\ell} \overline{Y_{\ell}(\alpha)} Y_{\ell}\left(\alpha^{\prime}\right),
$$

where, $a_{\ell}:=\frac{\tilde{a}_{\ell}}{4 \pi}, k=1$, and

$$
a_{\ell}=\frac{e^{2 i \delta_{\ell}}-1}{2 i}=e^{i \delta_{\ell}} \sin \delta_{\ell}
$$

and $\delta_{\ell}$ are the phase shifts. One has [N, formula (11.19)]:

$$
a_{\ell}=-\int_{0}^{\infty} d r u_{\ell}(r) q(r) \psi_{\ell}(r)
$$


where $u_{\ell}(r)=r j_{\ell}(r) \sim \sin \left(r-\frac{\ell \pi}{2}\right)$ as $r \rightarrow \infty, j_{\ell}(r)$ are the spherical Bessel functions, $j_{\ell}(r):=$ $\sqrt{\frac{\pi}{2 r}} J_{\ell+\frac{1}{2}}(r)$, and $\psi_{\ell}(r)$ is the solution to the equations:

$$
\psi_{\ell}^{\prime \prime}+k^{2} \psi_{\ell}-\frac{\ell(\ell+1)}{r^{2}} \psi_{\ell}-q(r) \psi_{\ell}=0
$$

with the asymptotics:

$$
\psi_{\ell} \sim \frac{e^{i \frac{\pi}{2}(\ell+1)}}{2}\left(e^{-i r}-e^{-i \pi \ell} S_{\ell} e^{i r}\right) \text { as } r \rightarrow \infty, \quad S_{\ell}:=\exp \left(2 i \delta_{\ell}\right) .
$$

The function $\psi_{\ell}$ solves (uniquely) the integral equation

$$
\psi_{\ell}(r)=u_{\ell}(r)+\int_{0}^{\infty} g_{\ell}(r, s) q(s) \psi_{\ell}(s) d s, \quad k=1
$$

where

$$
\begin{gathered}
g_{\ell}(r, s)=-u_{\ell}(r) w_{\ell}(s), \quad r<s ; \quad g_{\ell}(r, s)=g_{\ell}(s, r), \\
w_{\ell}(s):=i \sqrt{\frac{\pi s}{2}} H_{\ell+\frac{1}{2}}^{(1)}(s), \quad u_{\ell}(r)=\sqrt{\frac{\pi r}{2}} J_{\ell+\frac{1}{2}}(r),
\end{gathered}
$$

and $H_{\ell}^{(1)}$ is the Hankel function.

It is known [RK, p.407] that

$$
J_{\nu}(r) \sim\left(\frac{e r}{2 \nu}\right)^{\nu} \frac{1}{\sqrt{2 \pi \nu}}, \quad H_{\nu}^{(1)}(r) \sim-i \sqrt{\frac{2}{\pi \nu}}\left(\frac{e r}{2 \nu}\right)^{-\nu}, \quad J_{\nu}(r) H_{\nu}^{(1)}(r) \sim-\frac{i}{\pi \nu}, \quad \nu \rightarrow+\infty
$$

and [AR, Appendix 4]:

$$
\left|J_{\nu}(r) H_{\nu}^{(1)}(r)\right|<\left(\nu^{2}-\frac{1}{16}\right)^{-\frac{1}{4}}, \quad \nu>\frac{1}{4} .
$$

It follows from (3.7) that $u_{\ell}(r)$ does not have zeros on any fixed interval $(0, a]$ if $\ell$ is sufficiently large. Define

$$
v_{\ell}(r):=\frac{\psi_{\ell}(r)}{u_{\ell}(r)}
$$

Then (3.4) yields

$$
v_{\ell}(r)=1+\int_{0}^{a} \frac{g_{\ell}(r, s) u_{\ell}(s)}{u_{\ell}(r)} q(s) v_{\ell}(s) d s .
$$

From (3.5) and (3.7) one gets

$$
\begin{gathered}
g_{\ell}(r, s) \sim \frac{r}{2 \ell+1}\left(\frac{r}{s}\right)^{\ell}, \quad r<s, \quad \ell \rightarrow+\infty, \\
\frac{u_{\ell}(s)}{u_{\ell}(r)} \sim\left(\frac{s}{r}\right)^{\ell+1}, \quad \ell \rightarrow+\infty .
\end{gathered}
$$

Thus

$$
g_{\ell}(r, s) \frac{u_{\ell}(s)}{u_{\ell}(r)} \sim \frac{s}{2 \ell+1}
$$


This implies that for sufficiently large $\ell$ equation (3.10) has small kernel and therefore is uniquely solvable in $C(0, a)$ and one has

$$
\psi_{\ell}(r)=u_{\ell}(r)\left[1+O\left(\frac{1}{\ell}\right)\right] \text { as } \ell \rightarrow+\infty, \quad 0 \leq r \leq a
$$

uniformly with respect to $r \in[0, a]$.

Note that in the book $[\mathrm{N}]$ formula (12.180), which gives the asymptotic behavior of $S_{\ell}$ for large $\ell$, is misleading: the remainder in this formula is of order which is much greater, in general, than the order of the main term in this formula. That is why we had to find a different approach, which yielded formula (3.14).

From (3.3), (3.6), (3.7) and (3.14) one gets

$$
a_{\ell}=-\int_{0}^{\infty} d r q(r) u_{\ell}^{2}(r)\left[1+O\left(\frac{1}{\ell}\right)\right]=-\int_{0}^{a} d r q(r) r^{2} r^{2 \ell}\left[1+O\left(\frac{1}{\ell}\right)\right] \frac{1}{4 \ell+2}\left(\frac{e}{2 \ell+1}\right)^{2 \ell+1} .
$$

Therefore, using (1.11), one gets:

$$
\lim _{\ell \rightarrow \infty}\left(\frac{2 \ell+1}{e}\left|a_{\ell}\right|^{\frac{1}{2 \ell}}\right)=\lim _{\ell \rightarrow \infty}\left|\int_{0}^{a} d r q(r) r^{2} r^{2 \ell}\right|^{\frac{1}{2 \ell}}=a
$$

Theorem 1 is proved.

Remark 3.1. Since $\delta_{\ell} \rightarrow 0$ as $\ell \rightarrow+\infty$, and $\sin \delta_{\ell} \sim \delta_{\ell}$, $e^{i \delta_{\ell}} \sim 1$, as $\delta_{\ell} \rightarrow 0$, formulas (3.16) and (3.2) imply:

$$
\lim _{\ell \rightarrow \infty}\left(\frac{2 \ell+1}{e}\left|\delta_{\ell}\right|^{\frac{1}{2 \ell}}\right)=a,
$$

where $\delta_{\ell}$ is the phase shift at a fixed positive energy. This is formula $\left(1.10^{\prime}\right)$.

Acknowledgement: AGR thanks Dr. M.Klaus for a discussion. 


\section{REFERENCES}

[AR] De Alfaro, V., Regge, T., Potential Scattering, North Holland, Amsterdam, 1965.

[BE] Bateman, H., Erdelyi, A., Tables of Integral Transforms, McGraw Hill, New York, 1954.

[L] Levin, B., Distribution of Zeros of Entire Functions, Amer. Math. Soc., Providence RI, 1980, Translations of Math. Monographs N5.

[MP] Mikhlin, S., Proessdorf, S., Integral Operators, Springer Verlag, New York, 1968.

[N] Newton, R., Scattering Theory of Waves and Particles, Springer Verlag, New York, 1982.

[R1] Ramm, A.G., Multidimensional Inverse Scattering Problems, Longman/Wiley, New York, 1992.

[R2] Symmetry properties for scattering amplitudes and applications to inverse problems, J. Math. Anal. Appl. 156 (1991), 333-340.

[R3] Completeness of the products of solutions to PDE and uniqueness theorems in inverse scattering, Inverse problems 3 (1987), L77-L82.

[R4] _ Recovery of the potential from fixed energy scattering data, Inverse Problems 4 (1988), 877-886; 5 (1989), 255.

[R5] Multidimensional inverse problems and completeness of the products of solutions to PDE, J. Math. Anal. Appl. 1341 (1988), 211-253; 139 (1989), 302.

[R6] _ Property $C$ with constraints, Comptes rend. Acad. Sci., Paris 321 (1995), no. 11, 11413-1417.

[R7] Completeness of the products of solutions of PDE and inverse problems, Inverse Probl. 6 (1990), 643-664.

[RK] Ramm, A.G., Katsevich, A.I., The Radon Transform and Local Tomography, CRC Press, Boca Raton, 1996.

E-mail address: ramm@math.ksu.edu 